\title{
Research Paper: Psychoeducation on Improving Mental Health Literacy and Adjustment to Illness in Patients With Type 2 Diabetes: An Experimental Study
}

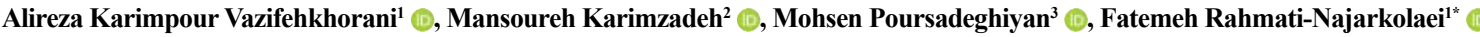 \\ 1. Health Research Center, Lifestyle Institute, Baqiyatallah University of Medical Sciences, Tehran, Iran. \\ 2. Pediatric Neurorehabilitation Research Center, University of Social Welfare and Rehabilitation Sciences, Tehran, Iran. \\ 3. Health Management and Economics Research Center, Iran University of Medical Sciences, Tehran, Iran.
}

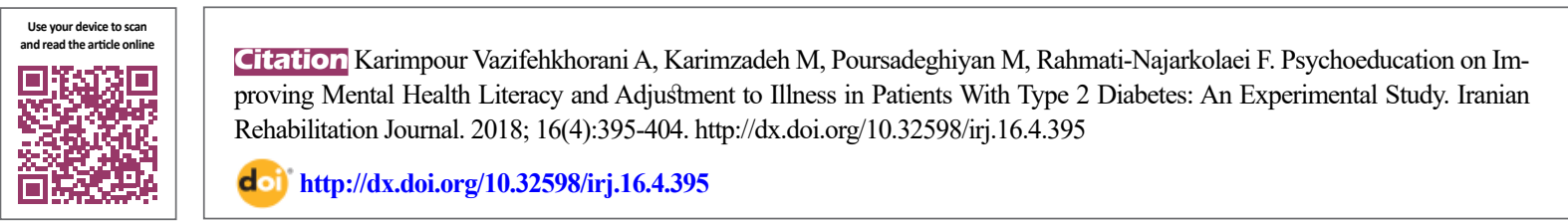

Article info:

Received: 10 Feb 2018

Accepted: 23 Jun 2018

Available Online: 01 Dec 2018

Keywords:

Cognitive Behavioral Therapy, Adjustment to illness, Mental health literacy, Type 2 diabetes

\section{A B STRACT}

Objectives: The present study investigated the role of psychoeducation in improving mental health literacy and adaptation in patients with type 2 diabetes.

Methods: The present study was an interventional study with pre-test, post-test and follow-up design with a control group. The study population included patients with type 2 diabetes who referred to Iran Hospital. Based on the research method and considering the missing data, a sample size of 80 people was considered. In total, 40 patients were selected as the intervention group and 40 subjects as the controls by purposeful sampling method. The subjects were randomly assigned into the two groups. The obtained data were collected by O'Connere and Casey's mental health literacy questionnaire and psychosocial compatibility questionnaire of Moro and colleagues. The purpose of psychological education in this study, which was used as the intervention, was Cognitive Behavioral Therapy (CBT). Training was performed in a group format. Therapeutic sessions were 11 one-hour sessions.

Results: The obtained results suggested that the effect of intervention in the post-test phase was $57 \%$ on mental health literacy and $48 \%$ in follow-up. In addition, the effect of intervention on adjustment in the post-test phase was $39 \%$, and in the follow-up phase $38 \%$.

Discussion: Psychological trainings like CBT can predict the information that is more important in diabetes self-management. Increasing the mental health literacy rate for psychiatric disorders associated with type 2 diabetes, improves compatibility, which will improve the quality of life and lifestyle of people with type 2 diabetes.

\section{* Corresponding Author:}

Fatemeh Rahmati-Najarkalaei, PhD.

Address: Health Research Center, Lifestyle Institute, Baqiyatallah University of Medical Sciences, Tehran, Iran.

Tel: +98 (21) 82482468

E-mail:fatemeh_rahmati@bmsu.ac.ir 


\section{Highlights}

- Cognitive behavioral therapy can predict the more important information in diabetes self-management, increase mental health literacy, and improves adaptability and quality of life.

- Cognitive behavioral models can improve beliefs and attitudes, and increase the sense of pain control, use of positive coping strategies, and reduction of negative emotional states in patients.

- Cognitive behavioral therapy can spot the role of cognitive factors in inefficient thoughts, underlying assumptions, and disordered information processing in diabetes pathology and the incidence of depression and compatibility problems in these patients.

\section{Plain Language Summary}

Diabetes is one of the prevalent diseases and impairs social, occupational and personal health of the patients. The common mental disorder among diabetic patients is depression. In recent years, one of the topics discussed in the prevention of psychological problems is mental health literacy that plays an important role in the management and control of negative effects of diabetes such as depression and adjustment disorders. For this reason, the focus of the present study is to improve the mental health literacy of patients with type 2 diabetes by psychological education (Cognitive behavioral therapy). The study results showed that cognitive behavioral therapy is effective in improving mental health literacy and adaptation to disease in patients with type 2 diabetes.

\section{Introduction}

iabetes is among the most common chronic and progressive metabolic diseases. It has a potentially harmful effect on the pancreas function of producing insulin and its use [1]. More than 220 million people worldwide suffer from diabetes [2]. Moreover, it is estimated that by 2050, the population of diabetics in the world will increase $165 \%$ [3]. More than three-quarters of whom live in developing countries [4]. This disease causes tension in individuals and affects their identity, psychosocial status, emotional balance, self-satisfaction, sense of competence and efficacy, social interactions and interpersonal relationships that require compatibility [5]. In fact, compatibility refers to the individual characteristics used by people for psychosocial management and improving their lives [6].

Compatibility with a chronic disease is a dynamic process, which is constantly affected by individual and environmental stimuli [7]. In other words, chronic diseases like diabetes challenge attitude towards life as a regular and continuous process, with significant psychological consequences [7]. Recent medical achievements have created this expectation in most patients and their families that all medical problems are curable. Thus, it is difficult for a person to accept the consequences of chronic illnesses and to use an appropriate coping strategy to treat his/her illness. Such condition causes psychosocial compatibility problems. In addition, the interdisciplinary approach of medicine has linked patients with more professionals. This situation increases the patients' expectations from themselves, despite their mental capacity limitations. Mental health issues like stress and anxiety have psychosomatic effects on human $[8,9]$.

This issue in some patients evokes the feeling of not being psychologically well supported. This may be a contributing factor to the formation of chronic diseases in a person $[7,10]$. Thus, a self-management approach is required in most chronic problems to control the disease [10]. Importantly, the psychological aspects of chronic diseases are often ignored. In other words, it is assumed that most patients are well adapted to the psychological aspects of chronic illnesses. However, compatibility is much difficult when experiencing a defect in physical health.

In general, about $20 \%$ to $25 \%$ of patients with chronic medical conditions experience significant psychological symptoms. Because of increasing self-care needs, chronic medical illnesses require behavioral changes [11]. Therefore, an effective management is the key to understanding the differences in disease patterns and their progression. Maintaining an independent and enjoyable life through effective psychological training and increased adaptability and mental health literacy are the 
main goals, rather than the treatment. Training also improves human behavior and cognition [12].

The psychological needs of people with chronic illnesses are internationally recognized. However, planned policies and cognitive behavioral services can meet the psychological needs of individuals including diabetic patients [11]. Providing independent living for diabetic patients by increasing the level of compatibility and mental health literacy relevant to these patients plays a significant role in their well-being. General knowledge about physical illnesses is very beneficial. However, providing information about mental disorders (mental health literacy) is somehow ignored [13]. For this reason, Jorm introduced the term mental health literacy and defined it as the knowledge and beliefs about mental disorders that help to identify, manage, and prevent mental disorders [14].

Mental health literacy includes a variety of components such as the ability to identify specific disorders or types of psychological distress, knowledge and beliefs about risk factors, causes, individual interventions/self-help, available specialized services, facilitating attitudes, appropriate identification and help seeking, and awareness about searching information about mental health. People experiencing disabling psychological symptoms or those who closely interact with people with these kinds of problems, try to manage those symptoms. The management of these symptoms is influenced by their mental health literacy. If successful, these management activities will reduce the disability symptoms and change the mental health literacy of individuals.

Maneze et al. reported that limited literacy is associated with poor health in various aspects [14]. This is especially true among the elderly, the minority, people with low education and those with chronic diseases, like type 2 diabetes. Diabetes is a chronic disease. Patients with low health literacy are less involved in understanding their illness and self-care aspects. Thus, they experience the worst health outcomes [15]. In addition, the literature review suggests that research on low mental health literacy in patients with type 2 diabetes is necessary.

Low mental health literacy among diabetic patients is associated with poor self-care and dysfunction in glycemic control (blood glucose control). The population of chronic diseases, including diabetes, is considered among high-risk groups. Psychoeducation can be used to reduce the complications and disabilities, and increase the level of compatibility and mental health literacy in this group. Psychoeducation involves interventions in which psychiatric and psychological educations can make changes in the behavioral and cognitive patterns of patients [12]

Educational interventions can play a preventive role at primary, secondary and tertiary prevention levels by helping the treatment process or reducing the disability caused by a disorder [16]. Accordingly, Cognitive Behavioral Therapy (CBT) can be effective in psychological problems in patients with type 2 diabetes. Cognitive behavioral education is among major treatments for chronic diseases. These treatments can have a coping, immunizing, and preventive effect on the development of various diseases and their complications by creating positive psychosocial impacts [16]. Therapeutic strategies of many medical centers in advanced countries are based on psychological and behavioral concepts like relaxation. In addition, cognitive methods of changing negative thinking are used as effective therapeutic strategies for the treatment of many disorders

This is because increasing information about diabetes does not always lead to improved self-care behaviors, better blood glucose control, and greater compatibility with the disease. Thus, when cognitive barriers related to attitude rather than the lack of knowledge or skills are the main obstacles of self-management, cognitive behavioral interventions are considered necessary. Such an approach is especially useful for patients who have repeatedly failed in their efforts to control diabetes.

Due to these experiences, such patients believe that they cannot effectively cope with the limitations and requirements of the treatment regimen. In other words, those with strongly negative beliefs about the effectiveness of their own control on diabetes are appropriate candidates for cognitive behavioral intervention [16]. Many studies have been conducted on diabetes and its comorbid disorders like depression. However, there are few studies in the field of mental health literacy and compatibility of these people to their illness. Therefore, the present study intended to examine the effectiveness of psychoeducation (CBT) on improving mental health literacy and compatibility of type 2 diabetic patients.

\section{Methods}

This was an interventional study with pre-test- posttest and follow-up design and a control group. The study population included patients with type 2 diabetes who referred to Iran Hospital. Based on the research method (in a pilot study, a sample size of at least 30 people per group was recommended), and considering the missing 
data, 80 subjects were selected by purposeful sampling method. In total, 40 patients were randomly assigned into the intervention group and 40 patients into the control group.

The inclusion criteria consisted of individuals with a diagnosis of type 2 diabetes for at least 3 years, physical symptoms of diabetes, deficits (but not disruptions) in personal and social performances due to diabetes, holding at least a high school diploma and the maximum age of 65 years. Exclusion criteria were reading and writing illiteracy, disagreement of participating in research and absence from a training session.

\section{Research tools}

The Mental Health Literacy Scale (MHLS): This scale was designed by O'Conner and Casey [17]. It includes 35 items with a Likert-type scale scoring. Its Cronbach alpha was reported 0.87 and its reliability using the test-retest method was reported 0.79 . In addition, in the present study, the Cronbach alpha coefficient was 0.89 and the retest coefficient for a 2 -week interval was 0.86 , which was performed on 90 patients with type 2 diabetes. Psychosocial Adjustment to Medical Illnesses Scale: This scale was developed by Morrow, Chiarello and Derogatis [18].

It contains 46 items and 7 subscales. The reliability and validity of its subscales respectively were health care orientation $(0.70 \& 0.46)$, social milieu $(0.72 \& 0.34)$, family environment $(0.52 \& 0.43)$, sexual relations $(0.81 \&$ $0.47)$, development of family relationships ( $0.33 \& 0.08)$, work environment $(0.62 \& 0.22)$, and mental distress $(0.82 \& 0.44)$. This scale was validated in Iran by Feghhi et al. [19], who reduced the original version to 45 questions. Feghhi et al. reported the Cronbach alpha of this scale equal to 0.94 , in patients with type 2 diabetes [19].

\section{Intervention method}

Psychoeducation, as the main purpose of the present study was performed though CBT. A cognitive behavioral therapy for depression [18] was applied in this study. However, its content was adjusted according to the variables of the present study. After determining the sample

Table 1. Summary of the Treatment Sessions Contents

\begin{tabular}{|c|c|}
\hline Session & Protocol \\
\hline $1^{\text {st }}$ & $\begin{array}{c}\text { Group member familiarity, group rules introduction, group members familiarity with the nature of their disease } \\
\text { and the role of psychological factors in the occurrence and exacerbation of symptoms, an introduction to CBT and } \\
\text { an explanation of diabetes-related mental disorders, an evaluation of members' expectations for participation in } \\
\text { the group, and relaxation training. }\end{array}$ \\
\hline $2^{\text {nd }} \& 3^{\text {rd }}$ & $\begin{array}{l}\text { Teaching cognitive patterns to patients, explaining spontaneous thoughts, providing record sheets of inefficient } \\
\text { thoughts, identifying the activating causes of disease symptoms and unpleasant emotions, examining potential prob- } \\
\text { lems in recording thoughts, identifying emotions, helping the patients with solving them, and relaxation training. }\end{array}$ \\
\hline $4^{\text {th }} \& 5^{\text {th }}$ & $\begin{array}{l}\text { Challenging irrational thoughts and beliefs, introducing challenges as strategies for coping with irrational thoughts } \\
\text { and beliefs, and ultimately changing them, facing a practical challenge with the irrational thoughts and beliefs that } \\
\text { the group members have recorded over the course of one week, profit and loss analysis and the Socratic question- } \\
\text { ing of thoughts that create unpleasant emotions and relaxation training. }\end{array}$ \\
\hline
\end{tabular}

Problem solving training, assertiveness training, activity planning training, discussing problem solving as a method of coping with worries, teaching three behavioral styles of passive, daring and aggressive in communication situations to strengthen self-expression and timing and planning training for doing activities and relaxation training.

$8^{\text {th }} \& 9^{\text {th }} \quad$ Familiarity with diabetes related mental disorders and their symptoms based on Diagnostic and Statistical Manual of Mental Disorders, $5^{\text {th }}$ Edition (DSM-5). patients' feedback about treatment sessions, performing a post-test. 
members, in order to perform the intervention, a pre-test was taken initially, using MHLS and Psychosocial Adjustment to Medical Illnesses Scale. Then, immediately after the intervention and training of psychological skills, a post-test was taken. One month later, trained patients performed a post-test as the follow-up to determine the effect of psychoeducation on improving mental health literacy. Education sessions were held in groups. In total, 11 one-hour sessions were held in an educational class in the hospital (Table 1).

\section{Data analysis}

To analyze the obtained data considering the method and objectives of the research, Multivariate Analysis of Covariance (MANCOVA) was used.

\section{Results}

In the present study, $47 \%$ (37 persons) of the participants aged 40 years and above, 23\% (19 persons) 35 40 years, $17 \%$ (14 persons) $30-35$ years and $12 \%$ (10 persons) $25-30$ years. In addition, $45 \%$ (36 persons) had high school diploma, 25\% (20 persons) had associate degree, 19\% (15 persons), 11\% (9 persons), and more.
Table 2 suggests that the average score of mental health literacy and adaptation to disease in the post-test and follow-up stages in the experimental group increased after the intervention, compared with the control group. In other words, psychoeducation was effective in improving mental health literacy and adaptation in patients with type 2 diabetes.

Box's Test was used to evaluate the equality of covariance matrices. The results of the test indicated that the correlation of dependent variables in the studied groups was homogeneous, because $\mathrm{F}(0.78)$ calculated at $\mathrm{P}>0.05$ was not significant. Thus, psychoeducation improved mental health literacy and adaptation in patients with type 2 diabetes (Table 3). The Levene's test was used to evaluate the equality of error or homogeneity of variancecovariance matrix. Table 4 indicates that the variation error of variables in the studied groups is homogeneous, because the calculated $\mathrm{F}$ is not significant at $\mathrm{P}<0.05$.

\section{Bonferroni test results}

Bonferroni post hoc test was used to compare the mean score of the mental health literacy and adaptation with disease variables. The results demonstrated that psychoeducation (intervention) significantly improved the men-

Table 2. Mean \pm SD of mental health literacy and adaptability variables

\begin{tabular}{llccccccc}
\hline & \multirow{2}{*}{ Variables } & \multicolumn{2}{c}{ Pre-Test } & \multicolumn{2}{c}{ Post-Test } & \multicolumn{2}{c}{ Follow-Up } \\
\cline { 3 - 8 } & & Mean & SD & Mean & SD & Mean & SD \\
\hline \multirow{3}{*}{ Intervention group } & Mental health literacy & 11.13 & 1.45 & 16.26 & 12.33 & 14.9 & 11.9 \\
& Adaptability to disease & 150.06 & 7.57 & 177.06 & 8.46 & 168.02 & 8.1 \\
\cline { 3 - 8 } Control group & Mental health literacy & 10.9 & 1.14 & 10.5 & 1.13 & 9.9 & 1.5 \\
& Adaptability to disease & 141.02 & 6.11 & 142.5 & 6.12 & 139.1 & 5.8 \\
\hline
\end{tabular}

Table 3. Box's test results

\begin{tabular}{cccc}
\hline Significance Level & $\mathbf{d f}^{\mathbf{2}}$ & $\mathbf{d f}^{\mathbf{1}^{2}}$ & $\mathbf{F}$ \\
\hline 0.73 & 72.08 & 21 & 0.78 \\
\hline
\end{tabular}

Table 4. Levene's test results based on the assumption of equality of variances in the two groups

\begin{tabular}{ccccc}
\hline Variables & $\mathbf{F}$ & $\mathbf{d f}^{\mathbf{1}}$ & $\mathbf{d f}^{\mathbf{2}}$ & Sig. \\
\hline Mental health literacy & 0.046 & 1 & 28 & 0.832 \\
Adaptability to disease & 0.109 & 1 & 28 & 0.743 \\
\hline
\end{tabular}


Table 5. ANCOVA results of pre-test, post-test and follow-up stages in the intervention and control groups

\begin{tabular}{lcccccccc}
\hline Variables & Stages & $\begin{array}{c}\text { Source of } \\
\text { Changes }\end{array}$ & TSS & df & MSE & F & Sig. & $\begin{array}{c}\text { Eta } \\
\text { Squared }\end{array}$ \\
\hline & \multirow{2}{*}{ Post-test } & Pre-test & 46.436 & 1 & 46.436 & 66.384 & 0.001 & 0.711 \\
$\begin{array}{c}\text { Mental health } \\
\text { literacy }\end{array}$ & & Intervention & 25.127 & 1 & 25.127 & 35.901 & 0.001 & 0.571 \\
& Follow-up & Pre-test & 1920.496 & 1 & 1920.496 & 971.565 & 0.001 & 0.973 \\
& & Intervention & 50.910 & 1 & 50.910 & 25.755 & 0.001 & 0.488 \\
& \multirow{2}{*}{ Post-test } & Pre-test & 269.200 & 1 & 269.200 & 241.210 & 0.001 & 0.899 \\
to disease & & Intervention & 19.692 & 1 & 19.692 & 17.644 & 0.001 & 0.395 \\
& \multirow{2}{*}{ Follow-up } & Pre-test & 240.610 & 1 & 240.610 & 389.411 & 0.001 & 0.735 \\
& & Intervention & 18.756 & 1 & 18.756 & 24.597 & 0.001 & 0.389 \\
\hline
\end{tabular}

Iranian Rehabilitation Journal

tal health literacy and adaptability in patients with type 2 diabetes. This is because after the intervention, their level enhanced significantly. The mental health literacy and adaptability with the mean score differences of 3.28 and 3.19, respectively were significant at $\mathrm{P}<0.01$.

According to Table 5, after adjusting the pre-test scores of the mental health literacy variable, the difference between the control and experimental groups was significant at $\mathrm{P}<0.001$. Therefore, psychoeducation for type 2 diabetic patients increases the mental health literacy in the experimental group compared with the control group in the post-test phase.

This intervention is $57 \%$ effective, that is, treatment intervention can explain $57 \%$ of the variance of total remaining scores. In addition, the effect of intervention in the follow-up phase was significant at $\mathrm{P}<0.001$, and the effect of this intervention was 48\%. Moreover, after modifying the pre-test scores in adjustment to disease variables, the difference between the control and experimental groups was significant at $\mathrm{P}<0.001$. In other words, psychoeducation improves compatibility in the experimental group compared to the control group in the post-test phase. This intervention is $39 \%$ effective, that is, the effect of intervention explain $39 \%$ of the variance of total remaining scores . In addition, the intervention in the follow-up phase was significant at $\mathrm{P}<0.001$ with an effect of $38 \%$.

\section{Discussion}

The obtained results revealed that psychoeducation (CBT) improved mental health literacy and adaptation in patients with type 2 diabetes at post-test and followup stages. This is because the effect of intervention in the post-test and follow-up on mental health literacy were $57 \%$ and $48 \%$, respectively. In addition, the effect of the intervention on the adaptation in the post-test was 39\% and it was $38 \%$ in the follow-up. Likewise, the results of Lorig study suggested that CBT diminished the level of disability and the use of medical services in chronic patients. It also optimized the level of social activity and improved the individuals' perception of their health and enhanced compatibility with a disease [20]. Michael suggested that psychosocial compatibility was among the most important variables in diabetes because it had a direct relationship with self-care behaviors.

A high level of compatibility is associated with better control of blood glucose in diabetic patients [21]. Moreover, Rapley concluded that psychosocial adjustment is the best predictor of self-care behaviors in type 2 diabetic patients [22]. Poor compatibility with a disease, using more services and poor outcomes of psychosocial compatibility with the disease is the strongest predictor of using services compared to other variables [22]. Furthermore, one of the predictors of adjustment in people with diabetes and in self-care behaviors is depression. In the research done by Lernmark et al. the depressed and non-depressed groups had a significant difference in metabolic control, adjustment, and self-esteem [23].

Snoek, Van der Ven et al. stated that people with very negative beliefs about the effectiveness of their own control on diabetes are appropriate candidates for CBT [24]. Davazdah Emamy et al. identified the effectiveness of stress management education through CBT to control blood glucose and depression in 20 patients with type 2 diabetes. It was concluded that the mean score of the blood glucose in the intervention group was significantly lower than that of the control group. They also reported the mean depression scores of them were significantly less than the controls after the intervention [25]. In addition, Peyrot observed that performing stress management training based on CBT on 23 women with diabetes 
for 12 sessions of 2 hours reduced the mean scores of depression, anxiety and stress in the intervention group compared to the controls [26].

The aforementioned studies are consistent with the results of present study indicating that CBT leads to increased adaptation. We also found that by providing specific information about psychological problems associated with diabetes, cognitive behavioral education increased the mental health literacy in type 2 diabetic patients. Furthermore, socio-cultural research that examined the factors affecting self-management of diabetes have been shown to be substantial in identifying the areas involved in completing cost-effective interventions.

In populations with cultural diversity associated with type 2 diabetes, although socio-cultural elements are determinants of health literacy, these factors are not part of health literacy. Whereas providing specific information about diabetes is more efficient in predicting behaviors associated with self-management of diabetes. In this regard, referring to the issue of incompatibility in perceiving health and the objective measurement of diabetes control in diabetes self-management education can improve patient's admission and supervision.

Depression is a significant factor in the prediction of low health literacy and a limited self-management of diabetes for professionals to screen depression. This way they can ensure that support is appropriate for people with type 2 diabetes, as it guarantees participation in the management of their own conditions. In other words, mental health illiteracy about diabetes and its associated psychiatric disorders can lead to compatibility problems. Jorm et al. introduced the term mental health literacy as the knowledge and beliefs about mental disorders that help to identify, manage and prevent them [13]. CBT can also be explained by considering the role of cognitive factors on inefficient thoughts, underlying assumptions, and disordered information processing in diabetes pathology and the incidence of depression and compatibility problems in these patients [16].

CBT attempts to identify irrational and ineffective thoughts and enables patients to realize the role of these thoughts in their illness and replace them with thoughts that are more correct. Patients with type 2 diabetes gradually develop helplessness and a sedentary lifestyle due to frequent exposures to negative events related to their illness. Isolation and inactivity also affect the patient's perception, and the set of these factors causes the feeling of inefficiency and helplessness in patients about their experiences. Indeed, the patient "loses many opportuni- ties for effective performance", leading to incompatibility in diabetic patients. In the present study, the patients' attitudes about the disease and its disabling effects were challenged by CBT. This included correcting automatic negative thoughts and underlying beliefs in patients. In addition, from the behavioral point of view, using the activity planning technique was very helpful.

Activities planning helps the patient to minimize the odds of failure in meeting daily schedule. In addition, patients are encouraged to value any increase in the level of daily activities and enhance positive thinking. Moreover, due to the nature of diabetes, its comorbid depression and highly irritable mood, most of these patients often develop difficulties in adapting and interpersonal relationships. Moreover, inappropriate communication styles have led to rejection by others, and a greater social isolation in these patients. Therefore, one of the goals of the treatment sessions was developing proper and assertive communications with self-expression in order to encourage the patients to establish appropriate social relationships and strengthen them [26].

According to Azami et al. diabetic patients fail to follow a proper diet. Their physical activity is improper, and their self-control activities are inadequate, leading to depression and compatibility disorders [27]. Therefore, CBT is beneficial to improve these patients' lifestyle [27]. Likewise, Petrak and Herperts suggested that CBT was a very effective treatment to increase the compatibility in such patients [28]. This is because cognitive behavioral models can improve beliefs and attitudes, and increase the sense of pain control, use of positive coping strategies and reduction of negative emotional states in patients [29]. In fact, cognitive behavioral methods are directly concentrated on "improving self-preventive behavior". These ways like this include such as apprenticeships or special tips for taking an action, preparing a calendar to remind the time of the intended behavior. Researchers have reported that these methods enhance the effectiveness of health promotion programs.

CBT is effective in increasing the compatibility of patients with type 2 diabetes. Moreover, because of providing specific information about psychiatric disorders associated with type 2 diabetes; it helps increase the mental health literacy in these patients [29]. To explain this, it can be said that CBT works on maladaptive schemas and misconceptions. Thus, it should provide cognitive reconstruction, predict more information on diabetes self-management, and increase mental health literacy associated with type 2 diabetes. Therefore, in the present research, 
this therapeutic approach improved the mental health literacy of individuals and increased their compatibility.

\section{Conclusion}

Generally, psychological training including CBT can predict the information that is important in diabetes selfmanagement. By increasing the mental health literacy about mental disorders associated with type 2 diabetes, the quality of life will improve in these patients. This helps them increase their compatibility with the disease.

One of the limitations of the present research was the use of self-monitoring tools for data collection. Additionally, the short-term follow-up phase is among the limitations of research. Therefore, it is suggested that other research tools be used to collect data in future research, and to conduct the follow-up study at least 2 months after the intervention. This study was also performed on patients with type 2 diabetes, which limits its generalizability. Thus, the findings must be generalized with caution.

\section{Ethical Considerations}

\section{Compliance with ethical guidelines}

The present study was approved by the Ethics Committee of the organization, (code No.: IR.bmsu. rec.1396.317).

\section{Funding}

This project was supported by Health Research Center, Lifestyle Institute, Baqiyatallah University of Medical Sciences, Tehran, Iran (code: Mhrc.95.604).

\section{Authors contributions}

The authors contributions is as follows: main investigator, designed the study, collected the data, performed analysis and wrote the first draft: Alireza Karimpour Vazifehkhorani; Supervision: Fatemeh Rahmati-Najarkolaei; study advisors: Mansoureh Karimzadeh and Mohsen Poursadeghiyan; and All authors read and approved the final revision of the manuscript.wer the study advisors.

\section{Conflict of interest}

The authors declared no conflict of interest.

\section{References}

[1] Olokoba AB, Obateru OA, Olokoba LB. Type 2 diabetes mellitus: A review of current trends. Oman Medical Journal. 2012; 27(4):269-73. [DOI:10.5001/omj.2012.68] [PMID] [PMCID]

[2] Fox CS, Golden SH, Anderson C, Bray GA, Burke LE, De Boer $\mathrm{IH}$, et al. Update on prevention of cardiovascular disease in adults with type 2 diabetes mellitus in light of recent evidence: A scientific statement from the American Heart Association and the American Diabetes Association. Circulation. 2015; 132(8):691-718. [DOI:10.1161/CIR.0000000000000230] [PMID]

[3] Stumvoll M, Goldstein BJ, van Haeften TW. Type 2 diabetes: Principles of pathogenesis and therapy. The Lancet. 2005; 365(9467):1333-46. [DOI:10.1016/S0140-6736(05)61032-X]

[4] Wild S, Roglic G, Green A, Sicree R, King H. Global prevalence of diabetes: Estimates for the year 2000 and projections for 2030. Diabetes Care. 2004; 27(5):1047-53. [DOI:10.2337/ diacare.27.5.1047] [PMID]

[5] Buus P, Lilleøre SK, Larsen K. Compatibility testing of two types of pen needles with a range of injection pens for diabetes medication. Current Medical Research and Opinion. 2011; 27(3):589-92. [DOI:10.1185/03007995.2010.547574] [PMID]

[6] Poursadeghiyan M, Omidi L, Hami M, Raei M, Biglari H. Drowsiness and its relation with individual characteristics among night workers in a desert hospital in Iran. International Journal of Tropical Medicine. 2016; 11(4):98-101.

[7] Norris SL, Engelgau MM, Narayan KV. Effectiveness of self-management training in type 2 diabetes: A systematic review of randomized controlled trials. Diabetes Care. 2001; 24(3):561-87. [DOI:10.2337/ diacare.24.3.561] [PMID]

[8] Poursadeghiyan M, Moghimian M, Amjad RN, Baneshi MM, Yari AR, Noroozi M, et al. Effects on job stress on Iranian clinical nurses. Annals of Tropical Medicine and Public Health. 2017; 10(4):985-8. [DOI:10.4103/ATMPH.ATMPH_306_17]

[9] Shahbazi A, Rahmani N, Abbasi M, Amjad RN, Marioryad $\mathrm{H}$, Khammar A, et al. Association between occupational stress and risk factors of cardiovascular disease in locomotive operators. Iranian Heart Journal. 2018; 19(2):20-6.

[10] Clark M, Hampson SE. Implementing a psychological intervention to improve lifestyle self-management in patients with type 2 diabetes. Patient Education and Counseling. 2001; 42(3):247-56. [DOI:10.1016/S0738-3991(00)00128-2]

[11] Protheroe J, Rowlands G, Bartlam B, Levin Zamir D. Health literacy, diabetes prevention, and self-management. Journal of Diabetes Research. 2017; 2017:1298315.

[12] Khandan M, Vosoughi S, Azrah K, Poursadeghiyan M, Khammar A. Decision making models and human factors: TOPSIS and Ergonomic Behaviors (TOPSIS-EB). Management Science Letters. 2017; 7(2):111-8. [DOI:10.5267/j. msl.2016.11.008]

[13] Jorm AF. Mental health literacy: Public knowledge and beliefs about mental disorders. The British Journal of Psychiatry. 2000; 177(5):396-401. [DOI:10.1192/bjp.177.5.396] [PMID]

[14] Maneze D, Everett B, Astorga C, Yogendran D, Salamonson $Y$. The influence of health literacy and depression on diabetes self-management: A cross-sectional study. Journal of Diabetes Research. 2016; 2016:3458969. 
[15] Ismail K, Winkley K, Rabe Hesketh S. Systematic review and meta-analysis of randomised controlled trials of psychological interventions to improve glycaemic control in patients with type 2 diabetes. The Lancet. 2004; 363(9421):1589-97. [DOI:10.1016/S0140-6736(04)16202-8]

[16] Egede LE, Ellis C, Grubaugh AL. The effect of depression on self-care behaviors and quality of care in a national sample of adults with diabetes. General Hospital Psychiatry. 2009; 31(5):422-7. [DOI:10.1016/j.genhosppsych.2009.06.007] [PMID]

[17] O'Connor M, Casey L. The Mental Health Literacy Scale (MHLS): A new scale-based measure of mental health literacy. Psychiatry Research. 2015; 229(1-2):511-6. [DOI:10.1016/j. psychres.2015.05.064] [PMID]

[18] Morrow GR, Chiarello RJ, Derogatis LR. A new scale for assessing patients' psychosocial adjustment to medical illness. Psychological Medicine. 1978; 8(4):605-10. [DOI:10.1017/ S003329170001881X] [PMID]

[19] Feghhi H, Saadatjoo S, Dastjerdi R, Kalantari S, Alidousti M. [The effect of a training program based on roy's adaptation model on psychosocial adaptation in patients with type II diabetes in Birjand, Iran (Persian)]. Jurnal of Zabol University of Medical Sciences and Health Services. 2014; 10(4):249-256.

[20] Lorig KR, Sobel DS, Stewart AL, Brown Jr BW, Bandura A, Ritter $P$, et al. Evidence suggesting that a chronic disease selfmanagement program can improve health status while reducing hospitalization: A randomized trial. Medical Care. 1999; 37(1):5-14. [DOI:10.1097/00005650-199901000-00003] [PMID]

[21] Michael SR. Integrating chronic illness into one's life: A phenomenological inquiry. Journal of Holistic Nursing. 1996; 14(3):251-67. [DOI:10.1177/089801019601400307] [PMID]

[22] Rapley P. Adapting to diabetes: Metabolic control and psychosocial variables. The Australian Journal of Advanced Nursing. 1990; 8(2):41-7.

[23] Lernmark B, Persson B, Fisher L, Rydelius PA. Symptoms of depression are important to psychological adaptation and metabolic control in children with diabetes mellitus. Diabetic Medicine. 1999; 16(1):14-22. [DOI:10.1046/j.14645491.1999.00008.x] [PMID]

[24] van Bastelaar KM, Pouwer F, Cuijpers P, Twisk JW, Snoek FJ. Web-based Cognitive Behavioural Therapy (W-CBT) for diabetes patients with co-morbid depression: Design of a randomised controlled trial. BMC Psychiatry. 2008; 8:9. [DOI:10.1186/1471-244X-8-9] [PMID] [PMCID]

[25] Davazdah Emamy M, Roshan R, Mehrabi A, Attari A. [The effectiveness of cognitive-behavioral stress management training on glycemic control and depression in patients with type 2 diabetes (Persian)]. Iranian Journal of Endocrinology and Metabolism. 2009; 11 (4):385-92.

[26] Peyrot M, Rubin RR. Behavioral and psychosocial interventions in diabetes: A conceptual review. Diabetes Care. 2007; 30(10):2433-40. [DOI:10.2337/dc07-1222] [PMID]

[27] Tol A, Azam K, Esmaeil Shahmirzadi S, Shojaeizadeh D, Mohebbi B, Asfia A, et al. [Relation between empowerment of diabetes control and adoption of self-management behaviors and its related factors among type 2 diabetic patients (Persian)]. Razi Journal of Medical Sciences. 2012; 19(98):11-18.
[28] Petrak F, Herpertz S. Treatment of depression in diabetes: An update. Current Opinion in Psychiatry. 2009; 22(2):211-7. [DOI:10.1097/YCO.0b013e3283207b45] [PMID]

[29] Sommaruga M, Spanevello A, Migliori GB, Neri M, Callegari S, Majani G. The effects of a cognitive behavioural intervention in asthmatic patients. Monaldi Archives for Chest Disease. 1995; 50(5):398-402. 
This Page Intentionally Left Blank 